\title{
Slicing the three-dimensional Ising model: Critical equilibrium and coarsening dynamics
}

\author{
Jeferson J. Arenzon,,$^{1, *}$ Leticia F. Cugliandolo, ${ }^{2, \dagger}$ and Marco Picco ${ }^{2, \ddagger}$ \\ ${ }^{1}$ Instituto de Física, Universidade Federal do Rio Grande do Sul, C.P. 15051, 91501-970 Porto Alegre, RS, Brazil \\ ${ }^{2}$ Sorbonne Universités, Université Pierre et Marie Curie-Paris VI, Laboratoire de Physique Théorique et Hautes Energies UMR 7589, \\ 4 Place Jussieu, 75252 Paris Cedex 05, France
}

(Received 28 December 2014; published 26 March 2015)

\begin{abstract}
We study the evolution of spin clusters on two-dimensional slices of the three-dimensional Ising model in contact with a heat bath after a sudden quench to a subcritical temperature. We analyze the evolution of some simple initial configurations, such as a sphere and a torus, of one phase embedded into the other, to confirm that their area disappears linearly with time and to establish the temperature dependence of the prefactor in each case. Two generic kinds of initial states are later used: equilibrium configurations either at infinite temperature or at the paramagnetic-ferromagnetic phase transition. We investigate the morphological domain structure of the coarsening configurations on two-dimensional slices of the three-dimensional system, compared with the behavior of the bidimensional model.
\end{abstract}

DOI: 10.1103/PhysRevE.91.032142

PACS number(s): 05.70.Ln, 64.60.Cn, 64.60.Ht

\section{INTRODUCTION}

Phase ordering kinetics is a phenomenon often encountered in nature. Systems with this kind of dynamics provide, possibly, the simplest realization of cooperative out-of-equilibrium dynamics at macroscopic scales. For such systems, the mechanisms whereby the relaxation takes place are usually well understood [1-3], but quantitative predictions of relevant observables are hard to derive analytically. Coarsening systems are important from a fundamental point of view, as they pose many technical questions that are also encountered in other macroscopic systems out of equilibrium that are not as well understood, such as glasses and active matter. They are also important from the standpoint of applications, as the macroscopic properties of many materials depend upon their domain morphology.

The hallmark of coarsening systems is dynamic scaling, that is, the fact that the morphological pattern of domains at earlier times looks statistically similar to the pattern at later times apart from the global change of scale [1-3]. Dynamic scaling has been successfully used to describe the dynamic structure factor measured with scattering methods, and the space-time correlations computed numerically in many models. It has also been shown in a few exactly solvable cases and within analytic approximations to coarse-grained models.

New experimental techniques now make possible the direct visualization of the domain structure of three-dimensional (3D) coarsening systems. In earlier studies, the domain structure was usually observed postmortem, and only on exposed two-dimensional (2D) slices of the samples, with optic or electronic microscopy. Nowadays, it has become possible to observe the full $3 \mathrm{D}$ microstructure in situ and in the course of evolution. These methods open the way to observation of microscopic processes that were far out of experimental reach. For instance, in the context of soft-matter systems, laser scanning confocal microscopy was applied to phase-separating binary liquids [4] and polymer blends [5-7],

\footnotetext{
*arenzon@if.ufrgs.br

${ }^{\dagger}$ leticia@1pthe.jussieu.fr

${ }^{\ddagger}$ picco@lpthe.jussieu.fr
}

while $\mathrm{x}$-ray tomography was used to observe phase-separating, glass-forming liquid mixtures [8] and the time evolution of foams towards the scaling state [9]. In the realm of magnetic systems the method presented in [10] looks very promising.

Three-dimensional images give, in principle, access to the complete topological characterization of interfaces via the calculation of quantities such as the Euler characteristics and the local mean and Gaussian curvatures. In addition to these very detailed analyses, one can also extract the evolution of the morphological domain structure on different planes across the samples and investigate to what extent the third dimension affects what occurs in strictly two dimensions. These in-plane studies are also relevant per se since in some case (e.g., metallic grains) there is an exposed surface to which experimental access is easy, even with simple optical techniques.

In this paper the focus is on the dynamic universality class of nonconserved scalar order parameter, as realized by Ising-like magnetic samples taken into the ferromagnetic phase across their second-order phase transition. An important question is to what extent the results for the morphological properties of strictly 2D coarsening apply to the 2D slices of 3D coarsening. In the theoretical study of phase ordering kinetics, a continuous coarse-grained description of the domain growth process, in the form of a time-dependent Ginzburg-Landau equation, is used. Within this approach, at zero temperature, the local velocity of any interface is proportional to its local mean curvature. Accordingly, in two dimensions the domains can neither merge nor disconnect in two (or more) components. In two dimensions one can further exploit the fact that the dynamics are curvature driven and use the Gauss-Bonnet theorem to find approximate expressions for several statistical and geometric properties that characterize the domain structure. In this way, expressions for the number density of domain areas, number density of perimeter lengths, relation between the area and the length of a domain, etc., were found [11,12]. In three dimensions, instead, the curvature-driven dynamics do not prohibit breaking a domain in two or merging two domains, and the Gauss-Bonnet theorem, which involves the Gaussian curvature instead of the mean curvature, cannot be used to derive expressions for the statistical and geometric properties of volumes and areas. Moreover, merging can occur in 2D slices 
of a 3D system, for example, via the escape of the opposite phase in-between into the perpendicular direction to the plane.

Two previous studies of 3D domain growth are worth mentioning here, although their focus was different from ours as we explain below. The morphology of the 3D zerotemperature nonconserved scalar order parameter coarsening was addressed in $[13,14]$. From the numerical solution of the time-dependent Ginzburg-Landau equation, results on the time dependence of the topological properties of the interfaces were obtained.

The late-time dynamics of the 3D Ising model evolving at zero temperature was analyzed in [15-18]. It was shown in these papers that the 3D Ising model (IM) does not reach the ground state or a frozen state at vanishing temperature. Instead, it continues to wander around an isoenergy subspace of phase space made of metastable states that differ from one another by the state of blinking spins that flip at no energy cost [15]. At very low temperatures the relaxation proceeds in two steps: first, with the formation of a metastable state similar to those of zero temperature and, next, with the actual approach to equilibrium [16]. The sponge-like nature of the metastable states was examined in [16-18].

In our study we use Monte Carlo simulations of the 3D IM on a cubic lattice with periodic boundary conditions, and we focus on the statistical and geometrical properties of the geometric domains and hull-enclosed areas on planes of the cubic lattice. A number of equilibrium critical properties, necessary to better understand our study of the coarsening dynamics in Sec. III, are first revisited in Sec. II. We start the study of the dynamics by comparing the contraction of a spherical domain immersed in the background of the opposite phase in $d=2$ and $d=3$, at both zero and finite temperature. This study, even though for a symmetric and isolated domain, allows us to evaluate the dynamic growing length and its temperature dependence. When the domains are not isolated, as is the case, for instance, of two circular slices lying on the same plane but being associated with the same 3D torus, merging may occur along evolution, and this process contributes to the complexity of the problem. We also study the dynamic scaling of the space-time correlation on the $2 \mathrm{D}$ slices. Following these introductory parts, the statistical and morphological properties of the areas and perimeters of geometric domains and hull-enclosed areas on 2D slices of the 3D IM are presented. We end by summarizing our results and by discussing some lines for future research in Sec. IV.

\section{THE MODEL AND ITS EQUILIBRIUM PROPERTIES}

Before approaching the dynamic problem we need to define the model and establish some of its equilibrium properties. This is the purpose of this section. The system sizes used in the equilibrium simulations range from $L=40$ to $L=800$ in $d=2$ and from $L=40$ to $L=400$ in $d=3$. The samples at the critical point were equilibrated with the usual cluster algorithms [19].

\section{A. The model}

The IM

$$
H_{J}=-J \sum_{\langle i j\rangle} s_{i} s_{j},
$$

with $s_{i} \pm 1, J>0$ and the sum running over nearest neighbors on a $d>1$ lattice, undergoes an equilibrium second-order phase transition at the Curie temperature $T_{c}>0$. The upper critical phase is paramagnetic and the lower critical phase is ferromagnetic.

In two dimensions the critical temperature $T_{c}$ coincides with the temperature at which the geometric clusters (a set of nearest-neighbor equally oriented spins) of the two phases percolate [20,21]. This is not the case in three dimensions: the percolation temperature, $T_{p}$, at which a geometric cluster of the minority phase percolates, is lower than the Curie temperature $T_{c}$ [22]. On the cubic lattice, which we use in this work, $T_{c} \simeq 4.5115$ [23] and $T_{p} \simeq 0.92 T_{c}$ [22] (a more recent determination yields $T_{p} \simeq 0.95 T_{c}$ [24]). The randomsite percolation threshold on the cubic lattice is $p_{c} \simeq 0.312$ [25]. The boundary conditions in the simulations are periodic.

\section{B. Equilibrium domain area distribution at $\boldsymbol{T}_{\boldsymbol{c}}$}

As already stated, the spin clusters in the 3D IM are not critical at the magnetic second-order phase transition [22]. Still, the 2D spin clusters on a slice, defined by taking all the spins in a 3D lattice $(x, y, z)$ with either $x, y$, or $z$ fixed, are critical with properties of a new universality class [26]. In particular, the distribution of the length of the surrounding interfaces, $N(\ell)$, and the area as a function of this length, $A(\ell)$, satisfy

$$
N(\ell) \simeq \ell^{-\tau_{\ell}}, \quad A(\ell) \simeq \ell^{\delta},
$$

with $\tau_{\ell} \simeq 2.23(1)$ and $\delta \simeq 1.23(1)$. Similar measurements were performed in Ref. [27], where a consistent value of the fractal dimension $d_{f}=2 / \delta$ was obtained. For the sake of comparison, the values of the exponents $\tau_{\ell}$ and $\delta$ for the critical spin clusters in two dimensions [12,28,29] are $\tau_{\ell}^{(2 \mathrm{D})}=27 / 11 \simeq 2.45455$ and $\delta^{(2 \mathrm{D})}=16 / 11 \simeq 1.45455=$ $\tau_{\ell}^{(2 \mathrm{D})}-1$.

As the main purpose of this paper is to characterize the out-of-equilibrium dynamics of the 3D IM by focusing on the behavior of the statistical and geometrical properties of the cluster areas on 2D slices, we further investigated the equilibrium properties of the same objects at the phase transition in two and three dimensions, with the purpose of checking whether the theoretical expectations are realized numerically for the system sizes we can simulate.

We consider the number density of cluster areas on a $2 \mathrm{D}$ plane. The area is defined as the number of spins in a geometric cluster that are connected (as first neighbors on the square lattice) to its border. With this definition one excludes the spins in the holes inside a cluster, that is, one considers the proper domain areas. At criticality, the number density of finite areas, excluding the percolating cluster, is given by the power law

$$
N(A) \simeq A^{-\tau_{A}} \text {. }
$$

The contribution of the percolating clusters can be included as an extra term that takes into account that these clusters should scale as $L^{2-(\beta / \nu)_{s}}$, where $(\beta / \nu)_{s}$ is the "magnetic exponent" associated with the spin clusters [25]. Thus, for a system of linear size $L$, the full distribution is

$$
\mathcal{N}(A)=L^{2} N(A)+a \delta\left(A-b L^{2-(\beta / v)_{s}}\right),
$$


and by construction, the normalization condition is

$$
\int d A A \mathcal{N}(A)=L^{2} \text {. }
$$

If the distribution $N(A)$ has a bounded support, with a maximum cluster of size $M_{L}$, then

$$
\int d A A \mathcal{N}(A) \simeq \frac{L^{2}}{\tau_{A}-2}\left(A_{0}^{2-\tau_{A}}-M_{L}^{2-\tau_{A}}\right)+a b L^{2-(\beta / v)_{s}},
$$

where $A_{0}$ is a microscopic area. The normalization condition can be satisfied only if

$$
M_{L} \simeq L^{-\frac{(\beta / v)_{s}}{2-\tau_{A}}}
$$

which, using a standard relation between exponents from percolation theory [25],

$$
(\beta / \nu)_{s}=d\left(\frac{\tau_{A}-2}{\tau_{A}-1}\right)
$$

yields

$$
M_{L} \simeq L^{2 /\left(\tau_{A}-1\right)} \simeq L^{2-(\beta / v)_{s}} .
$$

This implies that the largest size contributing to the distribution $N(A)$ has the same fractal dimension of the percolating cluster, thus scaling with the same power as the "magnetic term." The distribution of finite areas extends its support to a size-dependent size such that it matches the weight of the percolating clusters. In other words, there is no gap between the two contributions to Eq. (4). In the following, we first examine these relations in the 2D IM at its critical point and later come back to the slicing of the 3D systems.

\section{The critical $2 D I M$}

We first check the predictions listed above in the 2D IM at its critical point. In this case $(\beta / \nu)_{s}$ coincides with the magnetic exponent of the tricritical Potts model with $q=1$ [30]. Therefore,

$$
(\beta / \nu)_{s}^{(2 \mathrm{D})}=\frac{5}{96}, \quad \tau_{A}^{(2 d)}=\frac{379}{187},
$$

implying

$$
M_{L} \simeq L^{187 / 96}
$$

In Fig. 1 (inset) we present $A^{\tau_{A}} N(A)$ against $A / M_{L}$, with $N(A)$ the distribution of finite spin clusters, i.e., excluding the percolating cluster from each configuration. In this plot we used $M_{L} \simeq L^{2-(\beta / v)_{s}^{(2 D)}}$ with the exact value of $(\beta / \nu)_{s}^{(2 \mathrm{D})}$, and we determined the value of $\tau_{A}^{(2 \mathrm{D})}$ for each size $L$, finding that its dependence on $L$ is rather strong. It is only for the largest simulated system $(L=800)$ that $\tau_{A}^{(2 \mathrm{D})}$ becomes larger than 2 , converging, in the thermodynamical limit, to the right value, $379 / 187$, as shown in the inset in Fig. 2. Note, in the inset in Fig. 1, that $N(A)$ has a maximum for large clusters. These clusters are nonpercolating: in the overwhelming majority of cases, if the largest cluster percolates, thus contributing to the second term in $\mathcal{N}(A)$, the second largest does not and goes to $N(A)$. With the above exponents we obtain a nice scaling of this maximum in $N(A)$ as well as of the peak in $\mathcal{N}(A)$ (Fig. 1).

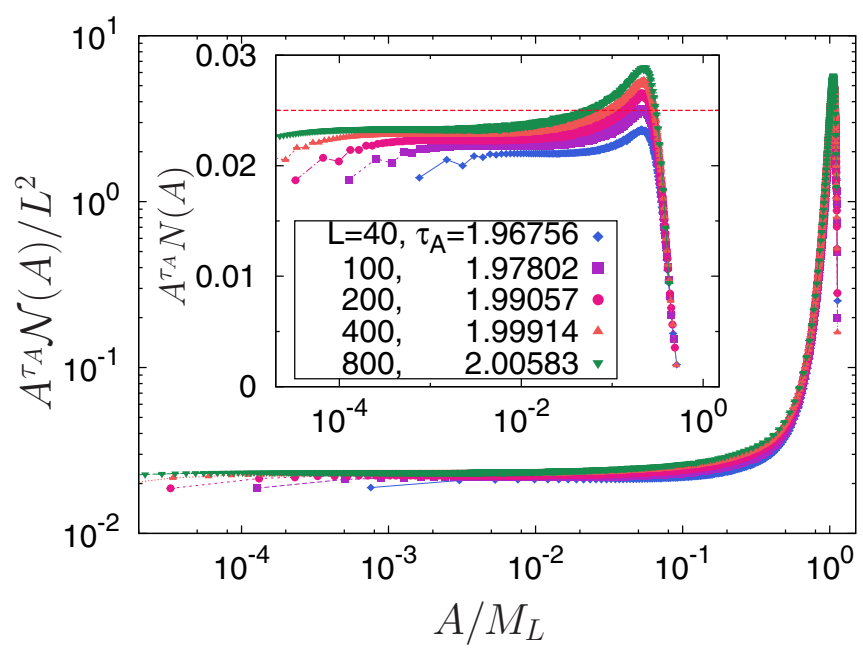

FIG. 1. (Color online) Distribution of the spin cluster sizes in the 2D IM at its critical temperature. Scaling of the number density of finite areas, $A^{\tau_{A}} N(A)$ (inset), and full distribution of all areas (main panel) vs $A / M_{L}$, with $M_{L}$ given by Eq. (11), for various system sizes $L$ given in the legend, together with the values of $\tau_{A}$ used. Inset: The dashed horizontal line is $c_{d} \simeq 0.025$ [12], to which the plateau should asymptotically converge.

The latter feature can be magnified by subtracting the support of finite clusters to leave only the areas of the percolating clusters. Indeed, by plotting $A^{\tau_{A}}\left[\mathcal{N}(A) / L^{2}-N(A)\right]$ as a function of $A / M_{L}$ (see Fig. 2), we obtain a perfect scaling. Note that the $\delta$ function appearing in Eq. (4) is for a single configuration. Different configurations differ in the value of $b$, originating the horizontal spread shown in the averaged distribution plotted in Fig. 2. Moreover, the plateau shown in the rescaled plot in the inset in Fig. 1 (dashed horizontal line), although smaller than the estimated value in Ref. [12],

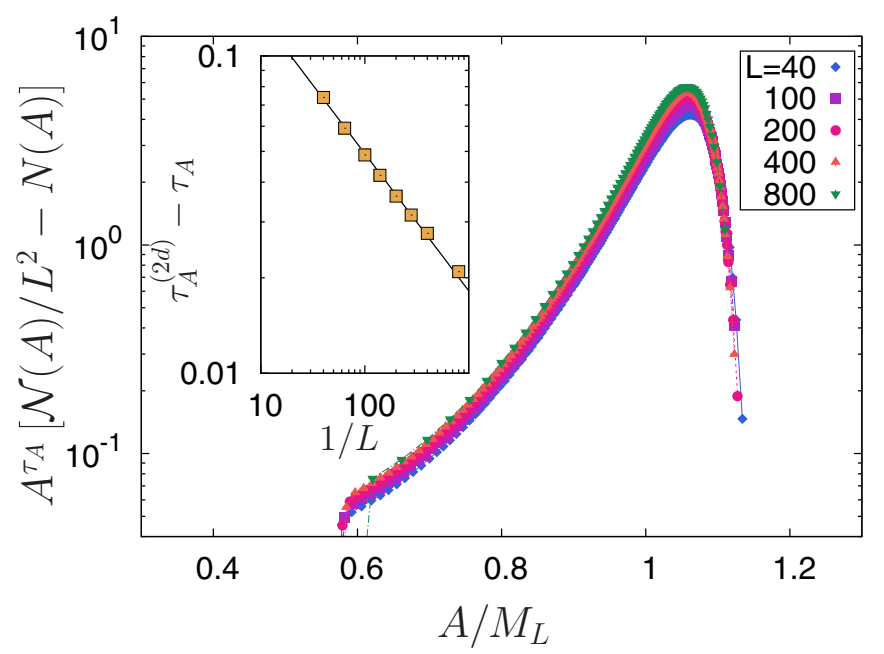

FIG. 2. (Color online) Distribution of the percolating spin cluster areas in the $2 \mathrm{D}$ IM at its critical temperature. Scaling of the number density of percolating areas, $A^{\tau_{A}}\left[\mathcal{N}(A) / L^{2}-N(A)\right]$, vs $A / M_{L}$, with $M_{L}$ given by Eq. (11), for various system sizes $L$ given in the legend. Inset: Values of $\tau_{A}$ from Fig. 1 as a function of $1 / L$ showing the convergence to the asymptotic value $\tau_{A}^{(2 \mathrm{D})}=379 / 187$. 
$c_{d} \simeq 0.025$, when extrapolated to very large sizes, is consistent with this value.

\section{Slicing the $3 D I M$}

Next we turn to the analysis of the domain areas on 2D slices of the critical 3D IM. The values of the exponents $(\beta / \nu)_{s}$ and $\tau_{A}$, and therefore the scaling of $M_{L}$ with $L$, are not known for these objects and we study them here. In Fig. 3 (inset) we show the distribution of the finite-size spin clusters, $N(A)$, and we determine $\tau_{A}$ for each size $L$. The measured values, shown in the legend, are much smaller than 2 even for the largest simulated system, and they seem to converge to a value of $\tau_{A} \simeq 1.94<2$. This fact is clearly disturbing since it implies that $M_{L}$ would decrease to 0 with increasing $L$. In Fig. 3 we rescale $A$ as $A / L^{x}$ and we obtain a good collapse of data for large $A$ with $x=1.86$. This implies that $M_{L} \simeq L^{1.86}$. In this figure, we also note that while the scaling of $N(A)$ is good for small values of the scaling variable, this is not the case for large ones. This fact is even clearer in Fig. 4, where we see that the part of the distribution that corresponds to the largest spin clusters does not scale. We observed that in some configurations the largest cluster does not percolate on the slice. The lack of scaling in Fig. 4 is probably related to the inconsistent value $\tau_{A}<2$ that we obtained from the analysis of $N(A)$.

There are two remarkable differences in $N(A)$ measured in the 2D system versus the sliced 3D one. The first is that the height of the plateau (dashed horizontal line in the inset in Fig. 3) seems to converge to a value that is about twice that found in the $2 \mathrm{D}$ case, that is, $2 c_{d} \simeq 0.05$ [12]. The second difference is that, in the slices, $N(A)$ does not present the maximum observed in two dimensions. This may explain why the distribution in this case is higher: the absence of a second, large cluster creates a large amount of space that will be filled by smaller ones.

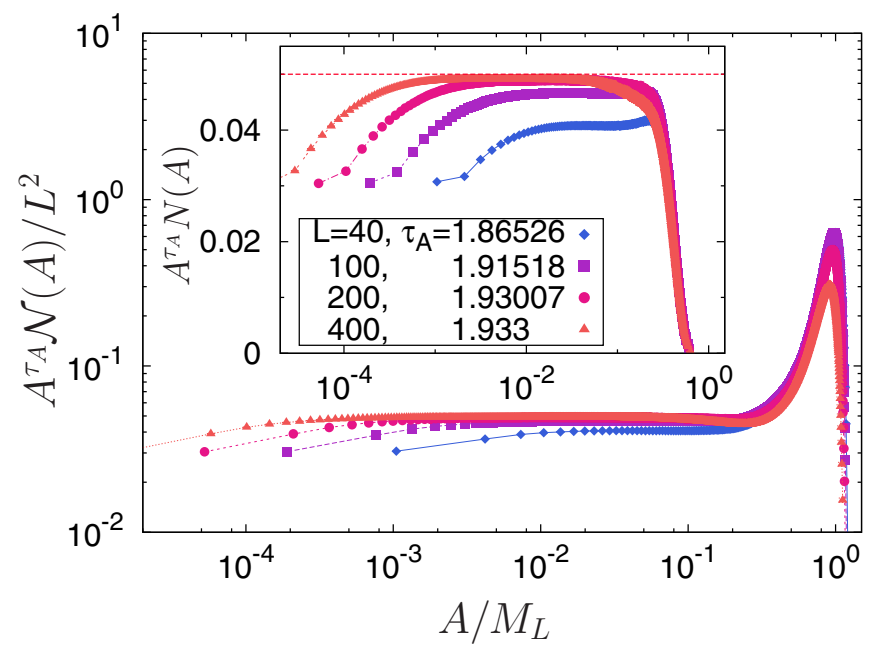

FIG. 3. (Color online) Distribution of spin cluster sizes for slices of the 3D IM at its critical temperature. Scaling of the number density of finite areas, $A^{\tau_{A}} N(A)$ (inset), and full distribution of all areas (main panel) vs $A / M_{L}$, with $M_{L} \simeq L^{1.86}$, for various system sizes $L$ given in the legend, together with the values of $\tau_{A}$ used. Inset: The dashed horizontal line is $2 c_{d} \simeq 0.05$ [12], twice the value for the $2 \mathrm{D}$ IM.

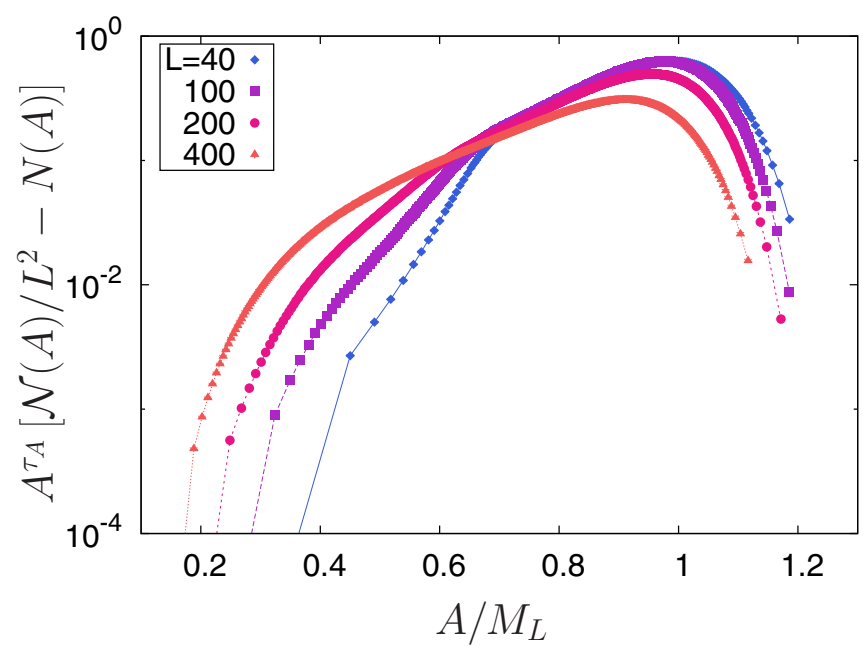

FIG. 4. (Color online) Distribution of the size of the largest cluster, $A^{\tau_{A}}\left[\mathcal{N}(A) / L^{2}-N(A)\right]$, vs $A / M_{L}$, with $M_{L} \sim L^{1.86}$ for spin cluster areas on slices of the $3 \mathrm{D}$ IM at its critical temperature. Differently from Fig. 2, the curves here do not scale.

We thus conclude that the small spin clusters living in slices of the 3D IM scale at the critical point, while the weight of the percolating clusters does not seem to. In order to clarify this issue we used the expected scaling of the largest cluster with the system size, $M_{L} \simeq L^{2-(\beta / v)_{s}}$, Eq. (9), to determine $(\beta / \nu)_{s}$. In Fig. 5, we show the values of the effective exponent $(\beta / \nu)_{s}$ obtained from a two-point fit,

$$
\left(\frac{\beta}{v}\right)_{s}\left(L, L^{\prime}\right)=2-\frac{\ln \left(M_{L} / M_{L^{\prime}}\right)}{\ln \left(L / L^{\prime}\right)},
$$

with $M_{L}$ the average size of the largest spin cluster. This quantity is expected to converge to a fixed value in the large size limit. However, for the sizes that we can simulate, it does not converge at the critical point. For the smallest sizes, $(\beta / v)_{s}\left(L, L^{\prime}\right)$ is close to a constant for $\beta=1 / T$ slightly

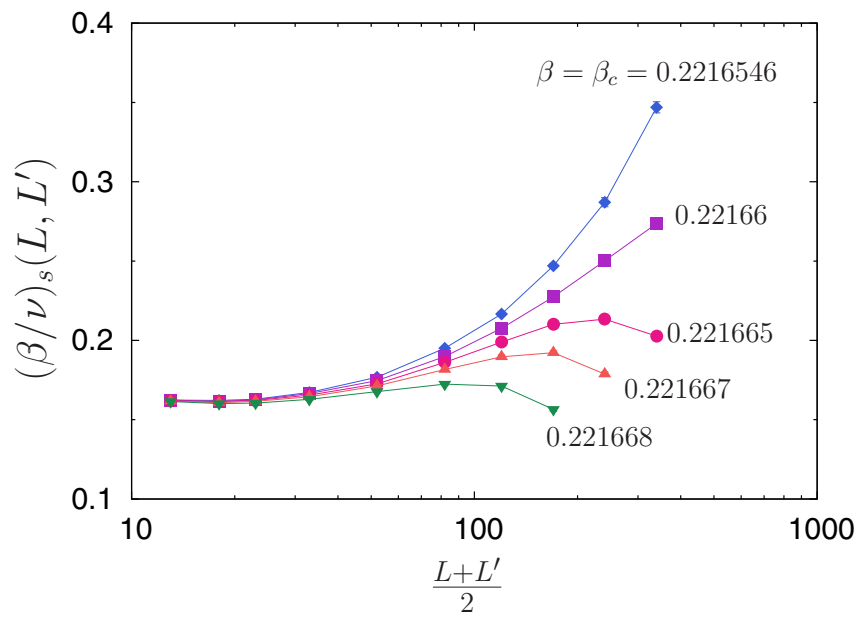

FIG. 5. (Color online) Effective value of $(\beta / v)_{s}\left(L, L^{\prime}\right)$ vs $(L+$ $\left.L^{\prime}\right) / 2$ for the $3 \mathrm{D}$ IM at its critical temperature as extracted from the analysis of the averaged size of the largest spin cluster on 2D slices; see Eq. (12). 
larger than $\beta_{c}$. Upon increasing the lattice size, we see that beyond some size, $(\beta / v)_{s}\left(L, L^{\prime}\right)$ drops. In the range $\beta_{c} \leqslant \beta<$ 0.221665 we do not reach an asymptotic regime and much larger sizes are needed to conclude the actual value of $(\beta / \nu)_{s}$. This also means that the values of $\tau_{A}$ that we computed can still increase and eventually become larger than 2 , as should happen. It is interesting to point out that we checked that the same analysis carried out on the Fortuin-Kasteleyn clusters obtained from the same data yield a value of $(\beta / \nu)_{s}$ in perfect agreement with the theoretical expectation.

We conclude that it is very hard to reach the asymptotic, large size limit in which the values of the exponents $(\beta / \nu)_{s}$ and $\tau_{A}$ for the areas of the geometric clusters on 2D slices of the $3 \mathrm{D}$ system should reach a stable limit.

\section{COARSENING PROPERTIES}

Once the system is prepared (equilibrated) at a specific temperature, it will be subcritically quenched and the outof-equilibrium subsequent dynamics studied. We start by presenting some background material on the $2 \mathrm{D}$ dynamics. We next describe the evolution of artificially designed singledomain initial states (circular or spherical in two or three dimensions, respectively, and a torus). After having analyzed these simple situations, the richer dynamics ensuing from an equilibrated state at $T_{0} \rightarrow \infty$ (noncritical) and $T_{0}=T_{c}$ (critical on the slices) are studied.

\section{A. Background}

With the coarse-grained approach, in two dimensions and in the absence of thermal fluctuations, one proves that the number of hull-enclosed areas per unit area, $n_{h}(A, t) d A$, with enclosed area in the interval $(A, A+d A)$, is $[11,12]$

$$
n_{h}(A, t)=\frac{(2) c_{h}}{\left(A+\lambda_{2 d} t\right)^{2}},
$$

where $c_{h}=1 /(8 \pi \sqrt{3})$ is a universal constant [31]. This result follows from the independent curvature-driven evolution of the individual hull-enclosed areas from initial values taken from a probability distribution determined by the initial state of the system. The statistics of the initial state is inherited in Eq. (13) by the factor in the numerator. Indeed, the factor 2 in parentheses is present when the initial state is prepared at $T>T_{c}$. It is due to the fact that the subcritical dynamics reach, after a time that grows with the system size as $t_{p} \simeq L^{\alpha_{p}}$, critical percolation [32]. Instead, it is absent if the initial state is one of the critical Ising point.

Temperature fluctuations have a double effect. On the one hand, their effect is incorporated in the factor $\lambda_{2 \mathrm{D}}$, which becomes $\lambda_{2 \mathrm{D}}(T)$ and takes into account the modification of the typical growing length (see below). On the other hand, small clusters are created by these fluctuations and the distribution Eq. (13) has to be complemented with an exponentially decaying term that takes into account the additional weight of thermal equilibrium domains.

The number density of the areas of the geometric domains cannot be derived exactly. Under some reasonable assump- tions, one argues [12] that at zero working temperature

$$
n_{d}(A, t)=\frac{(2) c_{d}\left(\lambda_{2 \mathrm{D}} t\right)^{\tau-2}}{\left(A+\lambda_{2 \mathrm{D}} t\right)^{\tau}},
$$

with the constant $c_{d} \simeq 0.025$ being very close to, albeit different from, $c_{h}$, and $\tau$ an exponent that takes the critical percolation or the critical Ising value, depending on whether the initial state is a high-temperature or a critical one.

The time dependence of these two number densities complies with dynamic scaling [1], with the typical length scaling as

$$
R(t) \simeq\left(\lambda_{2 \mathrm{D}} t\right)^{1 / 2} .
$$

As already said, the parameter $\lambda_{2 \mathrm{D}}$ is temperature, and material or model, dependent.

\section{B. Evolution of a single domain}

The coarse-grained domain growth process with nonconserved order parameter dynamics is described with a scalar field that follows a time-dependent Ginzburg-Landau equation [1]. From this equation, in the absence of thermal fluctuations, Allen and Cahn obtained a generic law that relates the local velocity of a point on an interface and the local mean curvature [33]

$$
v=-\frac{\lambda}{2 \pi} \kappa,
$$

with $\lambda$ a material-dependent parameter. The effect of temperature is usually incorporated into the prefactor [34-36], $\lambda(T)$.

In two dimensions, the area enclosed by a circle evolves in time as $\dot{A}=2 \pi R \dot{R}$. Under curvature-driven dynamics, the domain wall velocity, $v=\dot{R}$, is given by the Allen-Cahn law, (16). For the chosen geometry $\kappa=1 / R$ and the area of the disk decreases linearly in time, $\dot{A}=-\lambda_{2 \mathrm{D}}$, with a rate that is independent of $A$.

In three dimensions, the volume of a sphere evolves in time as $\dot{V}=4 \pi R^{2} \dot{R}$, the mean curvature is $\kappa=2 / R$, and the time variation of the volume is no longer independent of its size, $\dot{V}=-4 \lambda_{3 \mathrm{D}} R$. In three dimensions one can follow the surface area of the sphere, $A=4 \pi R^{2}$, and find $\dot{A}=-8 \lambda_{3 \mathrm{D}}$, or the area of the equatorial slice, $A=\pi R^{2}$, and find $\dot{A}=$ $-2 \lambda_{3 \mathrm{D}} \equiv-\lambda_{\mathrm{sl}}$.

We wish to check whether, and to what extent, these results remain valid on a cubic lattice with single-spin-flip dynamics. The fact that the area of an initial square or circular droplet in the 2D IM model with zero-temperature Glauber dynamics decreases to 0 linearly in time was proven in $[34,37,38]$. A rigorous bound, compatible with this time dependence, was derived in $[39,40]$ for the 3D IM with the same $T=0$ dynamics. In the rest of this section we analyze other initial states evolving at nonvanishing subcritical temperature.

\section{Single disk/sphere}

Here we simulate the IM starting from a configuration in which all spins that lie inside a circle in two dimensions or a spherical shell in three dimensions point up, while all other spins point down. This configuration is then allowed to evolve with Monte Carlo single-spin-flip dynamics. Figure 6 shows some snapshots at different times, with and without temperature fluctuations. 


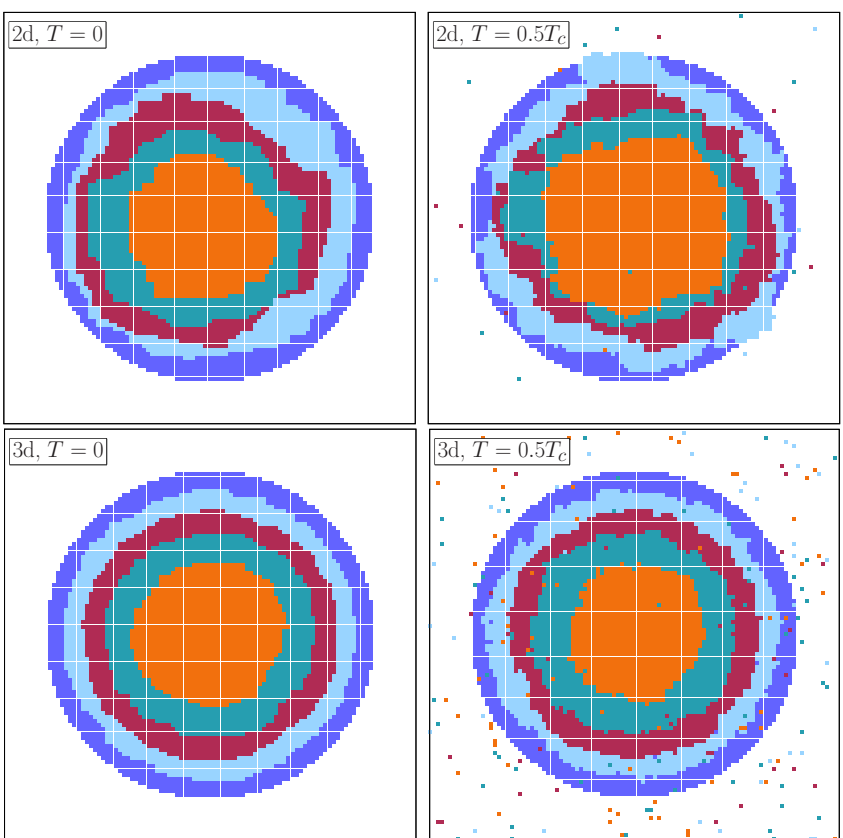

FIG. 6. (Color online) Time evolution of a 2D circular domain (top row) and an equatorial slice of a 3D spherical domain (bottom row) at $T=0$ (left column) and $T=T_{c} / 2$ (right column). In both cases the initial radius is $R_{0}=40$ (for finite temperatures, the box length must be large enough to prevent the circle from growing and percolating). Different colors correspond to different times (in Monte Carlo steps, starting from the background: $0,500, \ldots, 2500$ ). Although at zero temperature both areas decrease at the same rate, $\lambda_{\mathrm{sl}}(0)=\lambda_{2 \mathrm{D}}(0) \simeq 2$, in three dimensions the domains stay closer to their circular initial shape at all times.

By measuring how the size of the original bubble changes in time from the data gathered at zero temperature and shown in the left column, one verifies that the above relations for both $\dot{A}$ and $\dot{V}$ are satisfied at all times, with $\lambda_{2 \mathrm{D}}(0) \simeq 2$ (consistent with Ref. [34]) and $\lambda_{3 \mathrm{D}}(0) \simeq 1$, respectively. Note that, with these values, the product $\lambda \kappa$ is the same in two and three dimensions and $v=-(\pi R)^{-1}$. As a consequence, whatever the dimensionality, the radius behaves as

$$
R^{2}(t)=R_{0}^{2}-\frac{2}{\pi} t
$$

Therefore, an equatorial slice of the 3D sphere and the 2D disk should show the same behavior. Indeed, the area of the disk also decreases linearly in time with the same $T=0$ coefficient, $\lambda_{\mathrm{sl}}(0)=2 \lambda_{3 \mathrm{D}}(0)=\lambda_{2 \mathrm{D}}(0)=2$.

Interestingly, this value $\lambda_{\mathrm{sl}}=\lambda_{2 \mathrm{D}}=2$ is consistent with the average change, $\langle\dot{A}\rangle$, for a coarsening Ising system after having being quenched from an equilibrium state at either $T_{0} \rightarrow \infty$ or $T_{0}=T_{c}$ into the low-temperature phase, in which case the initial domains were no longer circular $[41,42]$. We conjecture that the fitting value $\lambda_{2 \mathrm{D}}(0) \simeq 2.1$, obtained in Refs. [11,12], is indeed exactly 2 .

Now turning the temperature on (data shown in the right column), we checked that

$$
\lambda_{\mathrm{sl}}(T) \simeq 2 \lambda_{3 \mathrm{D}}(T)
$$

at all temperatures.

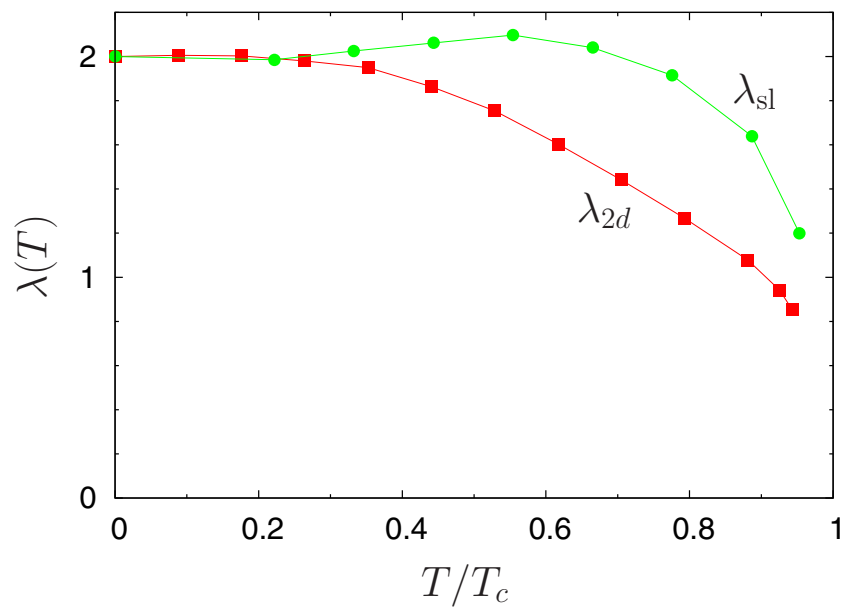

FIG. 7. (Color online) The parameter $\lambda(T)$, obtained from the shrinking of a single disk, $\lambda_{2 \mathrm{D}}$, or an equatorial slice of a single sphere, $\lambda_{\mathrm{sl}}$, as a function of the temperature.

A striking difference between the $2 \mathrm{D}$ and the $3 \mathrm{D}$ cases, both at zero and at nonvanishing temperature, is that the surface of the sliced 3D system stays closer to its original circular shape at all times, while the 2D system becomes more irregular. These surface fluctuations, stronger in two dimensions, are quite suppressed in three dimensions because of the extra surface tension along the direction orthogonal to the slice.

When the dynamics are affected by thermal noise, the behavior of $\lambda$ depends on the dimensionality, as shown in Fig. 7. Although $\lambda_{2 \mathrm{D}}(T)$, within our numerical precision, monotonically decreases as the temperature increases towards $T_{c}$ [34,43], this is not the case in three dimensions. Since the 3D system presents a large number of metastable states at $T=0$ [16-18], a small amount of noise may increase the wall velocity. Indeed, we find that $\lambda_{\mathrm{sl}}(T)$ has a maximum at intermediate temperatures. Nevertheless, although the temperature increases the roughness of the surface, the sliced disk still collapses more isotropically than the $2 d$ one, the fluctuations away from the circular shape being smaller. As the temperature approaches the critical value, $\lambda(T)$ tends to decrease to 0 in both cases. It is, however, very hard to conclude the exact $T$ dependence in this range by tracking the evolution of a single initial volume. Some of the sources of difficulties are the fragmentation and merging processes that occur because of the thermal fluctuations. Analogously, for the 3D case, isolated domains in the slice may belong to the same 3D cluster.

\section{Single toroidal domain}

In the continuous description of $2 \mathrm{D}$ coarsening the areas evolve independently of each other. Lattice effects do not affect this result at sufficiently large scales. However, although the dynamic mechanism in 2D slices of a 3D system is still curvature driven, the evolution of the areas on the slice may no longer be independent when, for instance, two areas on a slice do belong to the same 3D domain.

A simple initial configuration that illustrates the importance of the third dimension and the new mechanism that may arise on the slice is a toroidal structure. In Fig. 8 we show the time evolution of an initial toroidal domain observed on a plane 


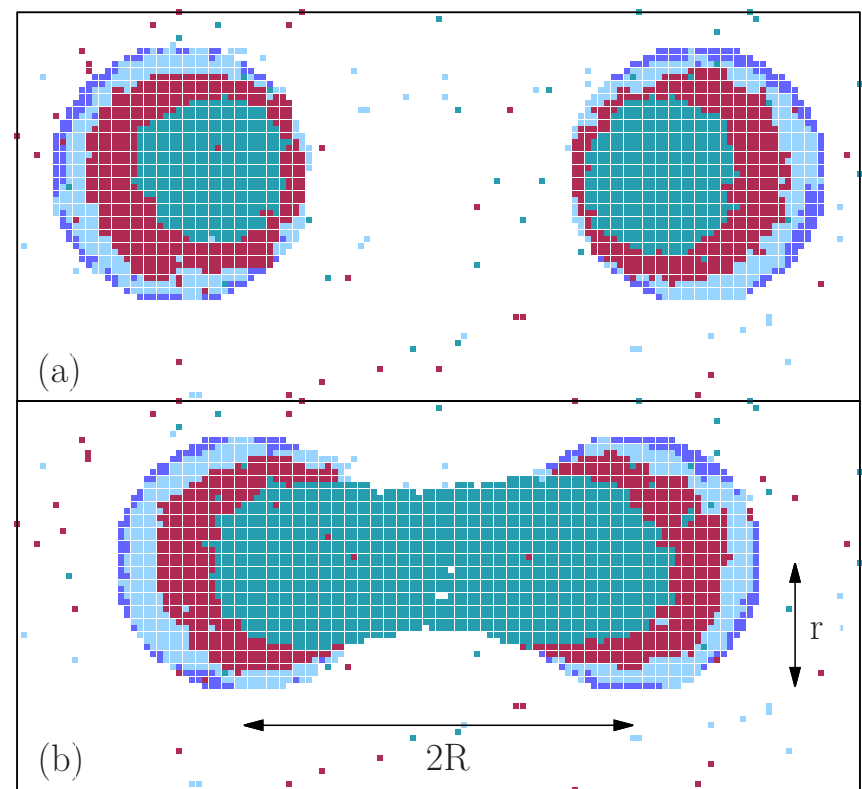

FIG. 8. (Color online) Evolution of a toroidal domain of one phase immersed in a sea of the opposite phase at $T=T_{c} / 2$ for two initial conditions. The snapshots of the cross section of a single-ring torus are shown with different colors at times $t=0,150,500$, and 1000 Monte Carlo steps (from back to front). Due to the shrinkage of the torus, there appears to be a small attraction between the domains as their centers get slightly closer with time. In both cases the minor radius is $r=20$ and, depending on the value of the major radius, $R=40$ (a) and $R=30$ (b), the two initially separated circles may merge. This merging mechanism, which slows down the change in area, is only present in the slices of a 3D system because the domains are connected along the orthogonal direction.

that contains its axis of revolution; that is, the initial state has two circular domains whose radii are the minor radius $r$ of the ring torus. The separation between their centers is twice the major radius $R$. In the two cases shown in the figure, the minor radius is the same, $r=20$, but the major radius is different: $R=40$ in Fig. 8(a) and $R=30$ in Fig. 8(b). The simulation is performed at $T_{c} / 2$. In both cases the whole toroid shrinks, and this can be seen as an effective attraction between the disks as they move towards each other. However, in the second case, the two initial disks change shape, and after some time, they merge and form an elongated domain in the plane. In the first case, the two disks do not merge on the observed time scale. Thus, differently from the pure $2 \mathrm{D}$ case where such a mechanism is absent, this merging process decreases the number of domains, increases the average area, and thus slows down the rate at which the average area decreases.

Whether or not the results for $\lambda$, shown in this section for the evolution of a single domain, transpose to the coarsening problem is analyzed below. Moreover, to what extent the above merging mechanism has an important role in this case is an open problem.

\section{Coarsening slices}

When the initial state, instead of being prepared as a single sphere immersed in a sea of opposite spins, is taken from the

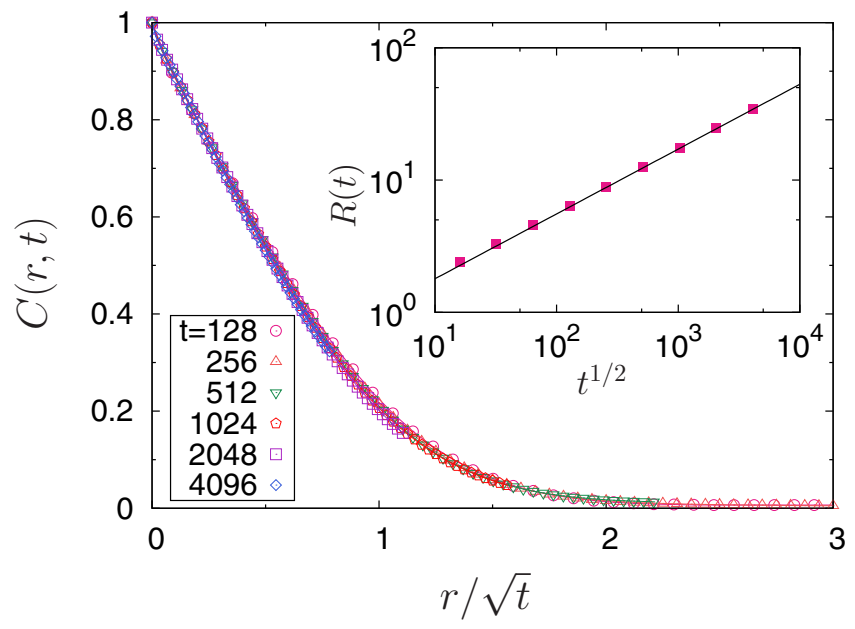

FIG. 9. (Color online) Collapsed equal time correlation $C(r, t)$, for several times after the quench from $T_{0} \rightarrow \infty$, indicated in the legend, as a function of the rescaled distance, $r / \sqrt{t}$. As expected, dynamical scaling is observed. Inset: length scale $R(t)$ obtained from $C(R, t)=1 / 2$. The straight line has exponent 0.5 .

equilibrium distribution at a given temperature above or at the critical point, much larger systems must be used in order to improve the statistics. Nonetheless, in three dimensions severe restrictions on the total size of the system are imposed. We here consider systems with a linear size up to $L=400$, and finite-size effects may still be important.

In two dimensions, the Ising model can be quenched to $T=0$ and yet evolve for a certain time before approaching either the ground state or a stripe state $[15,44]$, time that, in many cases, is enough to study coarsening phenomena [11,12]. In $d=3$, however, the $T=0$ dynamics get easily stuck in a sort of sponge state $[16-18,45]$. To avoid this halting of the configuration evolution, after the system is equilibrated either at $T_{0} \rightarrow \infty$ or at $T_{0}=T_{c} \simeq 4.51$, the quench is performed to a finite working temperature, $T=2$, well below $T_{p}<T_{c}$.

\section{1. $T_{0} \rightarrow \infty$}

We start the analysis by checking that dynamic scaling applies to correlation functions measured on the slices in the usual way. In Fig. 9 we display the equal-time correlation between spins at a distance $r$ on the slice, $C(r, t)$, as a function of the rescaled distance $r / t^{1 / 2}$, for several times given in the legend. The scaling is very satisfactory. In the inset we show the evaluation of the growing length scale $R(t)$ using the criteria $C(R, t)=1 / 2$; the straight line is the $t^{1 / 2}$ growth law of curvature-driven dynamics with a nonconserved order parameter. Note that although they could be taken into account, we neglect the corrections to scaling linked to the time scale $t_{p}$ discussed in Ref. [32], as they are not necessary for our purposes here.

At $T_{0} \rightarrow \infty$, the $2 \mathrm{D}$ slices of the $3 \mathrm{D}$ system are uncorrelated and any plane is statistically equivalent to a pure $2 \mathrm{D}$ system. Since both species of spins have, on average, the same density, no domain percolates along the slices (on the square lattice, $p_{c} \simeq 0.59$ [25]) and the distributions of areas and perimeters do not behave critically at $t=0[11,12]$ (see the corresponding 


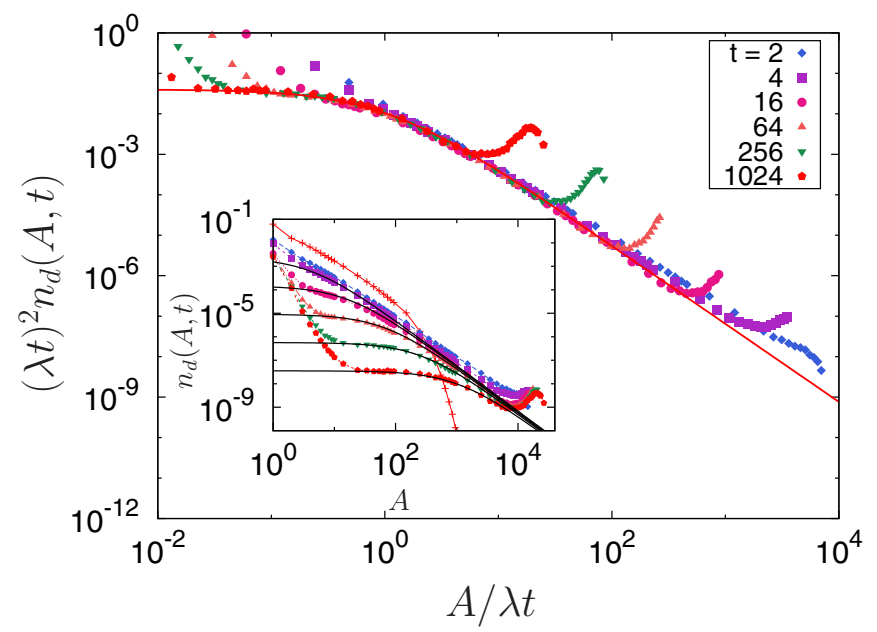

FIG. 10. (Color online) The number density of geometric domain areas (inset) and its rescaled form (main panel), in a 2D slice of the 3D IM (including the spanning clusters), per unit area of the system after a quench from $T_{0} \rightarrow \infty$ to $T=2$. Averages are over 7000 configurations (700 samples with 10 slices each) of an $N=200^{3}$ system. Note that since the linear system size is much smaller than the ones used in Ref. [11], the distributions have smaller cutoffs. Albeit the $3 \mathrm{D}$ system is far from the percolation threshold, the distributions on the slice soon approach a power-law distribution with an exponent that is compatible, asymptotically and for large systems, with $\tau_{p}=$ $187 / 91 \simeq 2.055$ (we use, indeed, the value obtained in Sec. II for $L=200: \tau_{A} \simeq 1.93$ ). The lines are the $2 \mathrm{D}$ result, Eq. (14), with $c_{d} \simeq$ $0.02, \lambda_{3 \mathrm{D}}(2) \simeq 1.04$, obtained from the single sphere, and the slope $\tau_{p}$ above. For small areas with respect to the typical one, $A / t<10$, the inset shows that the distribution of thermal fluctuations approaches its equilibrium form.

curve in the inset in Fig. 10). However, once quenched to a subcritical temperature, the critical state of the $2 \mathrm{D}$ site percolation is approached after a time that scales with the system size as $t_{p} \sim L^{\alpha_{p}}$. The exponent $\alpha_{p}$ is 0.5 on the square lattice [32] but we have not analyzed the scaling of $t_{p}$ for the $2 \mathrm{D}$ slices of the 3D system, which would constitute a project on its own. Nonetheless, since the phenomenology of both $2 \mathrm{D}$ and $3 \mathrm{D}$ slices is similar (the area distribution soon develops a power-law tail after the quench, as shown for $t=2$ and 4 in the inset in Fig. 10), we expect that $t_{p}$ will behave accordingly. In the whole $3 \mathrm{D}$ volume, on the other hand, since the random-site percolation threshold for the cubic lattice is $p_{c}=0.312$, there are percolating clusters of both species of spins at $t=0$. After the subcritical quench, the slices become correlated and the question we want to ask is to what extent the geometric properties, measured on a slice, resemble those of a $2 \mathrm{D}$ system.

As shown in both Figs. 10 and 11, the exponent of the power-law tail increases with time. Its asymptotic value, for geometric domains (Fig. 10), is consistent with the critical percolation value, $\tau_{p}=187 / 91 \simeq 2.055$ [25], although determining it precisely is a very hard task, as already discussed in Sec. II for the equilibrium data. For hull-enclosed areas (Fig. 11), on the other hand, the convergence to the asymptotic exponent (2) is fast. The distributions for geometric domains contain all clusters, even percolating ones, and present an

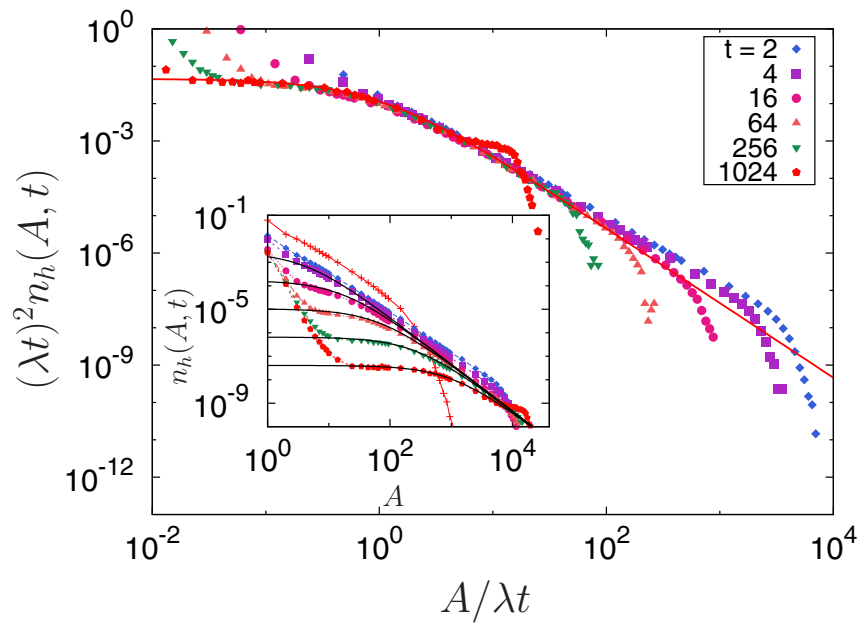

FIG. 11. (Color online) The same as Fig. 10, but for the hullenclosed areas. Differently from the geometric domains whose exponent $\tau_{A}$ has a strong size dependence, the power-law exponent here is 2 and is attained much more rapidly. The lines are the $2 \mathrm{D}$ result, Eq. (13), with $\lambda_{3 \mathrm{D}}(2) \simeq 1.04$, obtained from the single sphere. Note that for long times, the distribution develops a bump, even though the contribution from percolating clusters has been removed.

overshoot region that does not change position as the system evolves (and thus moves to the left when we rescale the areas by time, as shown in the figure). For the hull-enclosed areas there is no peak associated with the percolating domains (which are excluded by definition), but at later times the system develops a maximum anyway. The curves in these figures (inset) present, in the course of time, two other regimes. They all display a plateau, which crosses over to the power-law tail, and a first, very rapid decay in very small areas. The former is the actual curvature-driven regime. The latter are static and due to equilibrium temperature fluctuations. In Fig. 10 (inset) solid black lines represent the analytic law, Eq. (14), with $c_{d} \simeq 0.02$ and $\lambda(2) \simeq 1.04$. The constant $c_{d}$ takes the value used in Ref. [12] for the 2D case. The factor 2 in the numerator is associated with the high-temperature initial condition. The parameter $\lambda$ is very close in value to the one measured for the collapsing volume of a single sphere (see Sec. III B), evaluated at the working temperature $T=2$. Note that even though the measurements are done on a slice, the relevant coefficient is the one obtained for the whole volume of the sphere. Analogously, in Fig. 11 (inset) the lines are Eq. (13) with the same coefficient $\lambda_{3 \mathrm{D}}$ and $c_{h}=1 /(8 \pi \sqrt{3})$, again closely following the $2 \mathrm{D}$ results [11]. Upon rescaling the areas by time, as required by dynamic scale invariance, a rather good collapse of all curves onto a universal curve is found (see Figs. 10 and 11). As time increases, the power-law tail of the distributions is less visible (for these small system sizes).

Areas and perimeters are also correlated [12,42]. As an example, we present the collapsed curves (rescaling the area $A$ by $t$ and the perimeter $\ell$ by $t^{1 / 2}$ ) in Fig. 12 for the hull-enclosed areas and the corresponding perimeters (for geometric domains, the perimeter would also include the internal perimeters). Small domains are compact and round, thus $A \sim \ell^{2}$. Large domains are reminiscent of the large 


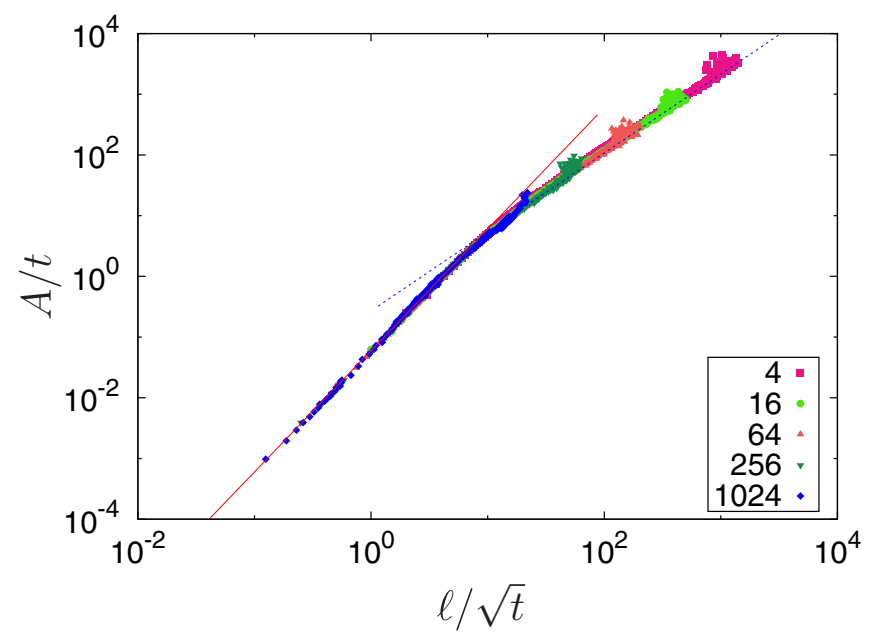

FIG. 12. (Color online) Rescaled hull-enclosed areas against the corresponding rescaled perimeters for slices of a 3D system quenched from $T_{0} \rightarrow \infty$ to $T=2$. For small sizes with respect to the typical one, since domains get round, the rescaled areas are simply the square of the rescaled lengths, $y \simeq x^{2}$. For large sizes, the rescaled quantities are linked by an exponent that is close to the one of critical 2D percolation, $8 / 7 \simeq 1.14[12,46]$. Numerically we find $y \simeq x^{1.2}$. Both behaviors, for small and large rescaled areas, are shown by straight dotted lines. The times at which the data are gathered are shown in the legend.

domains created soon after the quench, when the power law developed, and one expects that the area and perimeter are related as in critical percolation, $A \sim \ell^{8 / 7}$ [46]. Numerically, we find an exponent close to 1.2 , compatible with the $2 \mathrm{D}$ system value [12,42] and with the results in Ref. [26] for the equilibrium clusters. In summary, within the numerical precision of our simulation, the dynamical behavior on a slice of a 3D system is essentially equivalent to an actual $2 \mathrm{D}$ system when the initial state is prepared at $T_{0} \rightarrow \infty$. The surprise is that instead of using the value of $\lambda$ obtained from the measurements on a slice of the $3 \mathrm{D}$ sphere, $\lambda_{\mathrm{sl}}(T)$, the time-evolving distribution of geometric domains uses $\lambda_{3 \mathrm{D}}(T)$ related to the whole volume of the sphere, which is half of the previous one [see Eq. (18)].

\section{2. $T_{0}=T_{c}$}

As in the $T_{0} \rightarrow \infty$ case, we start the analysis by checking that dynamic scaling applies to correlation functions measured on the slices also in the case in which the quench is performed from $T_{0}=T_{c}$. We show in Fig. 13 the equal-time correlation between spins at a distance $r$ on the slice, $C(r, t)$, as a function of the rescaled distance $r / t^{1 / 2}$, for several times given in the legend. Once again, the scaling is good. Note also that the decay of the correlation keeps memory of the power-law present at the equilibrium state at $t=0, r^{2-d-\eta}$. Since the correlation is isotropic, measuring $C(r, t)$ on a slice or in the whole volume would give the same behavior, thus, in the power-law exponent, $d=3$ and $\eta=0.354$ (the value for the 3D Ising model). We present in the inset in Fig. 13 the growing length scale $R(t)$ extracted from $C(R, t)=1 / 2$ and the straight line $t^{1 / 2}$.

When prepared in an equilibrium state at the critical temperature $T_{0}=T_{c}$, several geometric distributions of the

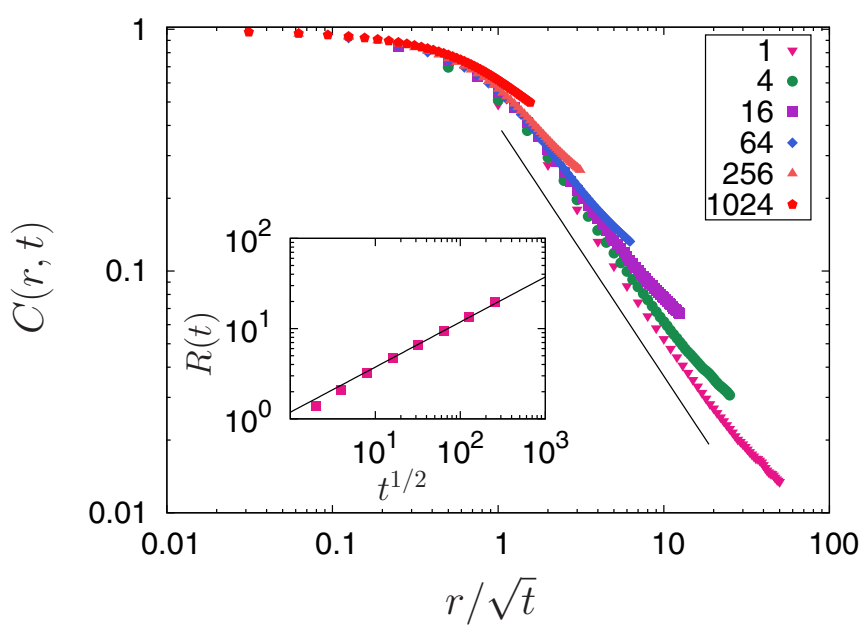

FIG. 13. (Color online) Collapsed equal time correlation $C(r, t)$ for several times after the quench from $T_{0}=T_{c}$, indicated in the legend, as a function of the rescaled distance, $r / \sqrt{t}$. As expected, dynamical scaling is observed. Inset: Length scale $R(t)$ obtained from $C(R, t)=1 / 2$. The straight line has exponent 0.5 .

2D system present power-law behavior since, in this case, the thermodynamical and the percolation transitions coincide. Although this is no longer the case in three dimensions, in which the percolation critical temperature associated with geometric domains is lower than the thermodynamical one, a 2D slice presents critical behavior at $T_{c}$, and as a consequence, one should find power-law behavior for several size distributions.

We saw in Sec. II that these distributions present strong finite-size effects, and in particular, the exponents are smaller than expected. For example, the known exponent for the distribution of geometric domain areas in the critical 2D $\mathrm{IM}$ is $\tau_{A}^{(2 \mathrm{D})}=379 / 187$ but this value is only approached asymptotically, for very large system sizes. For smaller rescaled sizes, the apparent exponent is even smaller than 2 , which would cause normalization issues. These effects are even stronger in a sliced 3D system, for which the data do not allow even a clear extrapolation of the exponent. Besides differing in the behavior of the exponent, the coefficient of the power-law distribution for a slice seems to have twice the value of the corresponding $2 \mathrm{D}$ distribution. It is thus interesting to see how these differences occurring at $t=0$ evolve after the system is quenched to a temperature lower than the critical one.

After the quench, there seems to be a very fast crossover to a distribution without the extra factor 2 in the coefficient [see Fig. 14 (inset)]. This is shown in the behavior of the distribution for small areas, as it approaches a constant value that does not depend on the exponent, only on the coefficient and the measuring time. Indeed, the curves in Fig. 14 (inset) are well fitted using Eq. (14) without the factor 2 in the coefficient. However, for large areas, the tail of the distribution is not well described, as one would expect, by Eq. (14) and the exponent measured at $t=0, \tau_{A} \simeq 1$.93. We must note, however, that the slices are small, thus the range of possible areas is rather limited. As time increases, the almost-flat part of the distribution gets wider and the power-law regime is hardly observed. In addition, the system suffers from finite-size 


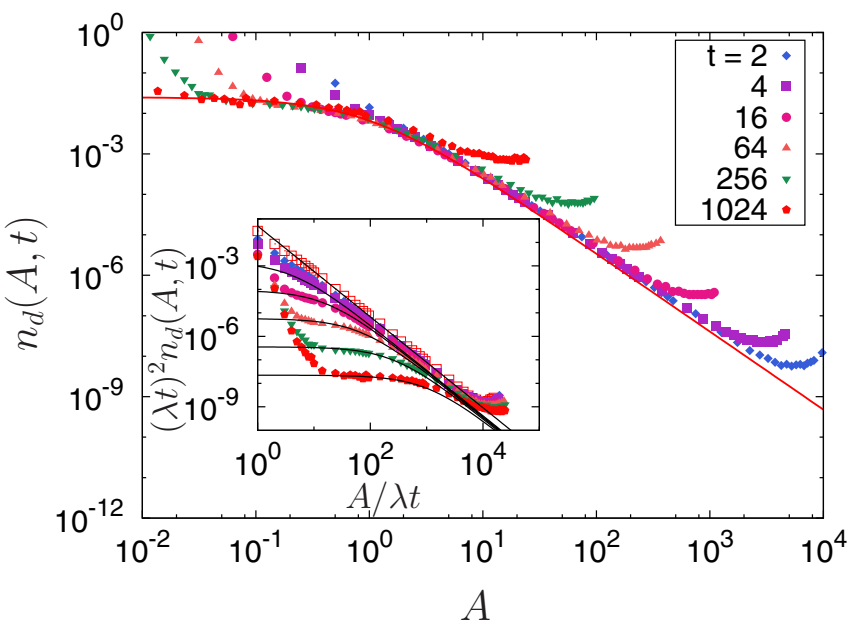

FIG. 14. (Color online) Number density of geometric domains per unit area after a quench to $T=2$ in a $2 \mathrm{D}$ slice of a $3 \mathrm{D}$ IM evolving from a $T_{0}=T_{c} \simeq 4.5115$ initial condition. Averages are over more than 7000 configurations. The solid line on top of the $t=0$ data (inset; open symbols) is $2 c_{d} / A^{1.93}$, while for $t>0$ we use Eq. (14) without the factor 2 in the coefficient. The main panel shows the collapsed version after proper rescaling of both axes. The solid line has the same exponent as the $t=0$ distribution but half the coefficient, $c_{d} / A^{1.93}$. Although the data could be well enveloped by a power law with an exponent slightly smaller than 1.93 , we remark that at later times, due to the small size of the slice, the power-law regime is barely observed in the simulation.

effects, even more severe than those for the 2D case as discussed in the previous section, and the observed $\tau_{A}$ does not even extrapolate to the right value. Nevertheless, we still observe the correct scaling as shown in Fig. 14. A similar

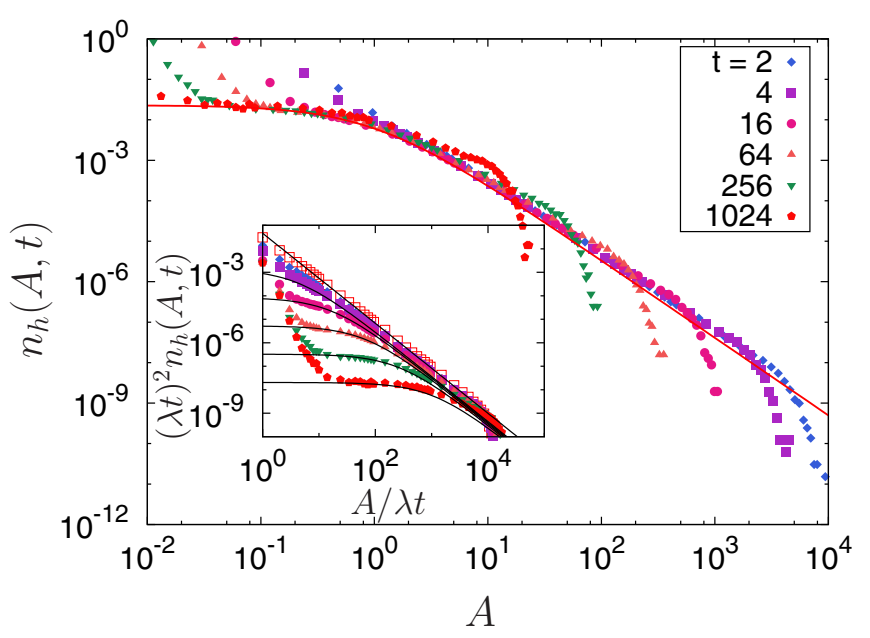

FIG. 15. (Color online) The same as Fig. 14, but for hullenclosed areas. The solid line on top of the $t=0$ data (inset; open symbols) is $2 c / A^{1.92}$, while for $t>0$ the distribution no longer has the factor 2 in the coefficient. The main panel shows the collapsed version after proper rescaling of both axes. Again, the solid line has the same exponent as the $t=0$ distribution but half the coefficient, $c_{d} / A^{1.92}$.

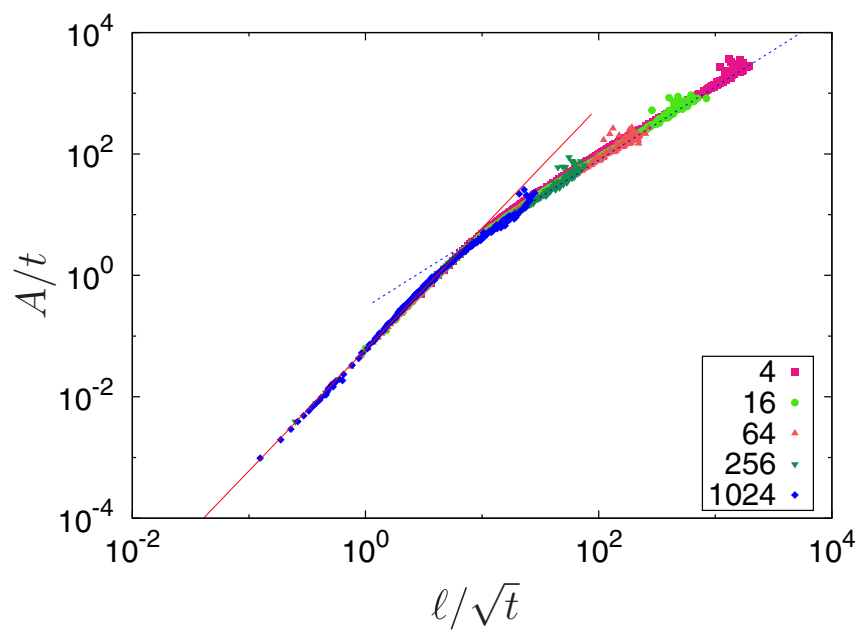

FIG. 16. (Color online) Rescaled hull-enclosed areas against the corresponding rescaled perimeters for slices of a 3D system quenched from $T_{0}=T_{c}$ to $T=2$. For small rescaled domains $y \sim x^{2}$ (dotted red line), while for larger ones $y \sim x^{1.3}$ (straight dotted blue line). This exponent is compatible with that for the geometric domains on $2 \mathrm{D}$ slices of the $3 \mathrm{D} \mathrm{IM}$ at equilibrium and $T_{c}, \delta \simeq 1.23$ [26], while it is well below the critical 2D exponent, $\delta^{(2 d)} \simeq 1.45$ [25] (dotted green line).

behavior, but with a slightly smaller exponent, is shown in Fig. 15 for hull-enclosed areas.

Areas and perimeters present, again, a two-regime relation. Small domains are round and $A \sim \ell^{2}$. This first regime can be observed in the small- $A$ part of Fig. 16, in which we relate the size of a hull with the area that it encloses. Larger domains, on the other hand, still encode some information on its original shape and deviate from the circular format. Indeed, roughly above $A / t \simeq 10$, the exponent decreases to 1.3 . This value is compatible with previous estimates [26] yet well below the critical $2 d$ exponent [29], 16/11 $\simeq 1.454$. Again, the origin for this discrepancy may be the strong finite-size effects previously discussed.

\section{CONCLUSIONS}

We have addressed the differences between clusters of a 2D slice of a 3D IM and culsters of an actual 2D system, both at equilibrium and while coarsening. We recall that the clusters on 2D slices of the 3D IM are critical at equilibrium at $T_{c}$ (contrary to the $3 \mathrm{D}$ structures). We have found that the distribution of finite clusters, $N(A)$, has a larger weight in the 2D slices than in the truly 2D model, whereas a second, very large (though still finite) cluster is mostly absent in the former while present in the latter. We have shown that, although we work with rather large system sizes, the measured exponents are still far from their asymptotic values when working on $2 \mathrm{D}$ slices.

Next we move to analysis of the geometric clusters and hullenclosed areas that develop after instantaneous quenches from equilibrium at the infinite and the critical temperatures. We found striking differences between the case with long-range correlations in the initial state $\left(T_{0}=T_{c}\right)$ and the case in which these do not exist $\left(T_{0}=\infty\right)$. In the absence of correlations, 
neighboring layers are independent, and even though strong correlations are built after a sudden subcritical quench, the subsequent behavior does not essentially differ (within our numerical precision) from that found in the strictly 2D case. On the other hand, for critical initial states, distant slices are correlated initially and this effect introduces differences between properties of the slices and properties of the actual $2 \mathrm{D}$ system. These differences already exist in the initial state, as explained in the previous paragraph. The extra weight in the finite-size areas (a factor of 2) seems to be washed out very rapidly after the quench and the small rescaled areas in the 2D slices soon become very similar (identical within our numerical accuracy) to those in the 2D system. Instead, the distribution and geometric properties of the large objects are much harder to characterize numerically in the $2 \mathrm{D}$ slices, as they are affected by strong finite-size effects. Although we find that the data satisfy dynamic scaling we cannot draw precise conclusions about the exponent characterizing the tail of the distribution or the area-perimeter law, as these are hard to determine numerically with good precision.

Work is in progress to extend these results to the 3D IM with order-parameter-conserving dynamics and to the Potts model.

\section{ACKNOWLEDGMENTS}

J.J.A. acknowledges the warm hospitality of the LPTHE (UPMC) in Paris during his stay where part of this work was done. J.J.A. was partially supported by the INCT-Sistemas Complexos and the Brazilian agencies CNPq, CAPES, and FAPERGS. L.F.C. is a member of Institut Universitaire de France.
[1] A. J. Bray, Adv. Phys. 43, 357 (1994).

[2] A. Onuki, Phase Transition Dynamics (Cambridge University Press, Cambridge, UK, 2004).

[3] S. Puri and V. Wadhawan (eds.), Kinetics of Phase Transitions (Taylor and Francis Group, London, 2009).

[4] W. R. White and P. Wiltzius, Phys. Rev. Lett. 75, 3012 (1995).

[5] H. Jinnai, Y. Nishikawa, T. Koga, and T. Hashimoto, Macromolecules 28, 4782 (1995).

[6] H. Jinnai, T. Koga, Y. Nishikawa, T. Hashimoto, and S. T. Hyde, Phys. Rev. Lett. 78, 2248 (1997).

[7] H. Jinnai, Y. Nishikawa, and T. Hashimoto, Phys. Rev. E 59, R2554 (1999).

[8] D. Bouttes, E. Gouillart, E. Boller, D. Dalmas, and D. Vandembroucq, Phys. Rev. Lett. 112, 245701 (2014).

[9] J. Lambert, R. Mokso, I. Cantat, P. Cloetens, J. A. Glazier, F. Graner, and R. Delannay, Phys. Rev. Lett. 104, 248304 (2010).

[10] I. Manke, N. Kardjilov, R. Schäfer, A. Hilger, M. Strobl, M. Dawson, C. Grünzweig, G. Behr, M. Hentschel, C. David et al., Nat. Commun. 1, 125 (2010).

[11] J. J. Arenzon, A. J. Bray, L. F. Cugliandolo, and A. Sicilia, Phys. Rev. Lett. 98, 145701 (2007).

[12] A. Sicilia, J. J. Arenzon, A. J. Bray, and L. F. Cugliandolo, Phys. Rev. E 76, 061116 (2007).

[13] M. Fialkowski, A. Aksimentiev, and R. Holyst, Phys. Rev. Lett. 86, 240 (2001).

[14] M. Fialkowski and R. Holyst, Phys. Rev. E 66, 046121 (2002).

[15] V. Spirin, P. L. Krapivsky, and S. Redner, Phys. Rev. E 63, 036118 (2001).

[16] V. Spirin, P. L. Krapivsky, and S. Redner, Phys. Rev. E 65, 016119 (2001).

[17] J. Olejarz, P. L. Krapivsky, and S. Redner, Phys. Rev. E 83, 051104 (2011).

[18] J. Olejarz, P. L. Krapivsky, and S. Redner, Phys. Rev. E 83, 030104 (2011).

[19] M. Newman and G. Barkema, Monte Carlo Methods in Statistical Physics (Oxford University Press, New York, 1999).

[20] K. Binder, Ann. Phys. 98, 390 (1976).

[21] A. Coniglio, C. R. Nappi, F. Peruggi, and L. Russo, J. Phys. A: Math. Gen. 10, 205 (1977).

[22] H. Müller-Krumbhaar, Phys. Lett. A 50, 27 (1974).
[23] A. L. Talapov and H. W. J. Blöte, J. Phys. A 29, 5727 (1996).

[24] A. R. de la Rocha, P. M. C. de Oliveira, and J. J. Arenzon [Phys. Rev. E (to be published)], arXiv:1502.05596.

[25] D. Stauffer and A. Aharony, Introduction To Percolation Theory (Taylor and Francis, London, 1994).

[26] V. S. Dotsenko, M. Picco, P. Windey, G. Harris, E. Martinec, and E. Marinari, Nucl. Phys. B 448, 577 (1995).

[27] A. A. Saberi and H. Dashti-Naserabadi, Europhys. Lett. 92, 67005 (2010).

[28] J. L. Cambier and M. Nauenberg, Phys. Rev. B 34, 8071 (1986).

[29] C. Vanderzande and A. L. Stella, J. Phys. A: Math. Gen. 22, L445 (1989).

[30] A. L. Stella and C. Vanderzande, Phys. Rev. Lett. 62, 1067 (1989).

[31] J. Cardy and R. M. Ziff, J. Stat. Phys. 110, 1 (2003).

[32] T. Blanchard, F. Corberi, L. F. Cugliandolo, and M. Picco, Europhys. Lett. 106, 66001 (2014).

[33] S. M. Allen and J. W. Cahn, Acta Metal. 27, 1085 (1979).

[34] S. A. Safran, P. S. Sahni, and G. S. Grest, Phys. Rev. B 28, 2693 (1983).

[35] K. A. Fichthorn and W. H. Weinberg, Phys. Rev. B 46, 13702 (1992).

[36] M.-D. Lacasse, M. Grant, and J. Viñals, Phys. Rev. B 48, 3661 (1993).

[37] D. Kandel and E. Domany, J. Stat. Phys. 58, 685 (1990).

[38] L. Chayes, R. H. Schonmann, and G. Swindle, J. Stat. Phys. 79, 821 (1995).

[39] P. Caputo, F. Martinelli, F. Simenhaus, and F. L. Toninelli, Commun. Pure Appl. Math. 64, 778 (2011).

[40] H. Lacoin, Commun. Math. Phys. 318, 291 (2013).

[41] M. P. O. Loureiro, J. J. Arenzon, L. F. Cugliandolo, and A. Sicilia, Phys. Rev. E 81, 021129 (2010).

[42] M. P. O. Loureiro, J. J. Arenzon, and L. F. Cugliandolo, Phys. Rev. E 85, 021135 (2012).

[43] M. Grant and J. D. Gunton, Phys. Rev. B 28, 5496 (1983).

[44] T. Blanchard and M. Picco, Phys. Rev. E 88, 032131 (2013).

[45] F. Corberi, E. Lippiello, and M. Zannetti, Phys. Rev. E 78, 011109 (2008)

[46] H. Saleur and B. Duplantier, Phys. Rev. Lett. 58, 2325 (1987). 